\title{
Normal Motor Adaptation in Cervical Dystonia: A Fundamental Cerebellar Computation is Intact
}

\author{
Anna Sadnicka • Bansi Patani - Tabish A. Saifee • Panagiotis Kassavetis • \\ Isabel Pareés • Prasad Korlipara • Kailash P. Bhatia • John C. Rothwell • \\ Joseph M. Galea $\cdot$ Mark J. Edwards
}

Published online: 29 May 2014

(C) The Author(s) 2014. This article is published with open access at Springerlink.com

\begin{abstract}
The potential role of the cerebellum in the pathophysiology of dystonia has become a focus of recent research. However, direct evidence for a cerebellar contribution in humans with dystonia is difficult to obtain. We examined motor adaptation, a test of cerebellar function, in 20 subjects with primary cervical dystonia and an equal number of aged matched controls. Adaptation to both visuomotor (distorting visual feedback by $30^{\circ}$ ) and forcefield (applying a velocitydependent force) conditions were tested. Our hypothesis was that cerebellar abnormalities observed in dystonia research would translate into deficits of cerebellar adaptation. We also examined the relationship between adaptation and dystonic head tremor as many primary tremor models implicate the cerebellothalamocortical network which is specifically tested by this motor paradigm. Rates of adaptation (learning) in cervical dystonia were identical to healthy controls in both visuomotor and forcefield tasks. Furthermore, the ability to adapt was not clearly related to clinical features of dystonic head tremor. We have shown that a key motor control function of the cerebellum is intact in the most common form of primary dystonia. These results have important implications for current anatomical models of the pathophysiology of dystonia. It is important to attempt to progress from general statements that implicate the cerebellum to a more specific evidence-based model. The role of the cerebellum in this enigmatic disease perhaps remains to be proven.
\end{abstract}

\footnotetext{
A. Sadnicka $\cdot$ B. Patani - T. A. Saifee $\cdot$ P. Kassavetis $\cdot$ I. Pareés $\cdot$ P. Korlipara · K. P. Bhatia · J. C. Rothwell · J. M. Galea $(\bowtie) \cdot$ M. J. Edwards

Sobell Department for Motor Neuroscience, UCL Institute of Neurology, 33 Queen Square, London WC1N 3BG, UK e-mail: joe.galea.01@gmail.com
}

J. M. Galea

School of Psychology, University of Birmingham, Birmingham, UK
Keywords Spasmodic torticollis $\cdot$ Cerebellum $\cdot$ Forcefield adaptation $\cdot$ Visuomotor adaptation $\cdot$ Motor control

\section{Introduction}

Primary dystonia is an enigmatic disease, which since its original description in 1911 has provoked lively debate surrounding its definition and pathophysiology [1,2]. For many years dystonia was conceptualised as a basal ganglia disorder; however, recently, a case has been made for cerebellar involvement within a growing dystonic sensorimotor network $[3,4]$. In rodent models modulating cerebellar function can cause or abolish dystonia (4-6). In humans, pathology of the cerebellum can produce secondary dystonia and there is a growing literature linking cerebellar dysfunction to primary dystonia (7). However, this area of research is still at an early phase and defining the extent and nature of cerebellar involvement in patients with primary dystonia is incomplete.

In the motor control literature, the archetypal cerebellardependent paradigm is adaptation. This paradigm requires subjects to adapt their performance of a task (such as reaching to hit a target) after an environmental perturbation (such as distortion of visual feedback) introduces a movement error. The sensory prediction error (how the actual sensory movement outcome differed from the predicted sensory movement outcome) is used to update subsequent motor performance, with this type of learning being strongly dependent on the cerebellum [5]. For example, the ability to adapt behaviour in response to a novel perturbation is impaired in patients with cerebellar lesions [6-8]. It is thought that the cerebellum is crucial for the formation of forward models, which predict the sensory consequences of a motor command and drive adaptation [9]. Thus, the ability to adapt has direct relevance to the clinical manifestation of dystonia and if impaired would 
provide a valuable model of how the cerebellum contributes to the pathophysiology of dystonia.

We have examined the ability to adapt as a marker of cerebellar function in 20 subjects with primary cervical dystonia and an equal number of aged matched controls. A purpose built robotic arm enabled detailed kinematic analysis of arm movements, and we have tested adaptation to both visuomotor and forcefield perturbations for which visual and proprioceptive afferent feedback dominate, respectively $[10$, 11]. Our hypothesis was that cerebellar abnormalities observed in dystonia research would translate into deficits of cerebellar adaptation. We also examined the relationship between adaptation and dystonic head tremor as many primary tremor models implicate the cerebellothalamocortical network which is specifically tested by this motor paradigm [12].

\section{Methods}

\section{Subjects}

Twenty patients with primary cervical dystonia were recruited from the National Hospital for Neurology and Neurosurgery, London (Table 1). Patients were tested at least 3 months after their last botulinum toxin treatment, and none were taking oral medications for dystonia. Twenty age-matched controls were also recruited. Subjects did not have any additional neurological or musculoskeletal problems of the arm or significant cognitive impairment. Written informed consent was obtained from all participants. The study had been approved by the local ethics committee.

\section{Clinical Assessment}

Severity of cervical dystonia was examined using the Toronto Western Spasmodic Torticollis Rating Scale (TWSTRS). Head tremor was objectively captured by tri-axial accelerometry prior to the adaptation task with a commodity mobile communication device (HTC Desire) at a sampling frequency of $100 \mathrm{~Hz}$ and analysed off-line. The device was strapped to the head below the occipital protuberance. Tremor recordings were made for $30 \mathrm{~s}$. Data were analysed with Spike software (CED electronics, version 2). The accelerometry axis with the greatest overall amplitude was used for subsequent analysis. A high pass Butterworth filter (corner 2) was applied and then a Fourier transform of the signal was derived. The dominant frequency was determined by the peak of the frequency spectrum. Total power of the spectra between 1 and $30 \mathrm{~Hz}$ was used as a marker of tremor severity.

Robotic Apparatus and Task

Participants were seated with their forehead supported on a headrest. Their semipronated right hand gripped a
Table 1 Clinical characteristics of patients. The severity subscore of the TWSTRS is out of 35 . The total TWSTRS which also incorporates disability and pain subscores is out of a total of 87

\begin{tabular}{|c|c|c|c|c|c|}
\hline \multirow[t]{2}{*}{ Age } & \multirow[t]{2}{*}{ Symptomatic head tremor? } & \multicolumn{2}{|l|}{ TWSTRS } & \multicolumn{2}{|l|}{ Tremor } \\
\hline & & Severity subscore & Total & Frequency $(\mathrm{Hz})$ & Power \\
\hline 56 & Yes & 6 & 38 & NA & NA \\
\hline 76 & Yes & 2 & 12 & NA & NA \\
\hline 68 & Yes & 24 & 44 & 5.8 & 85.68 \\
\hline 53 & No & 18 & 27 & 5.6 & 2.11 \\
\hline 61 & No & 15 & 23 & 6.6 & 0.08 \\
\hline 75 & Yes & 2 & 11 & 3.1 & 17.78 \\
\hline 63 & No & 2 & 14 & 4.8 & 0.08 \\
\hline 39 & No & 13 & 16 & 6.7 & 2.40 \\
\hline 40 & No & 19 & 40 & 4.3 & 0.08 \\
\hline 40 & No & 20 & 43 & 4.9 & 0.11 \\
\hline 61 & Yes & 16 & 44 & 5.5 & 14.37 \\
\hline 66 & No & 8 & 16 & 7.1 & 1.07 \\
\hline 69 & Yes & 17 & 35 & 3.5 & 1.02 \\
\hline 71 & Yes & 18 & 61 & 3.7 & 0.39 \\
\hline 80 & Yes & 9 & 44 & 3.8 & 2.83 \\
\hline 57 & Yes & 20 & 24 & 4.1 & 2.14 \\
\hline 51 & Yes & 24 & 56 & 7.5 & 2.48 \\
\hline 67 & Yes & 16 & 21 & 3.5 & 7.20 \\
\hline 53 & Yes & 11 & 27 & 4.9 & 5.59 \\
\hline
\end{tabular}


manipulandum underneath a horizontally suspended mirror. The mirror prevented direct vision of the hand and arm and showed a reflection of a computer monitor mounted above. The visual display comprised of a central $30 \mathrm{~mm}$ square which indicated the starting position, a circular cursor $(5 \mathrm{~mm}$ diameter) representing the position of the manipulandum and a $10 \mathrm{~mm}$ square target at one of four radially arranged positions $\left(45^{\circ}, 135^{\circ}, 225^{\circ}\right.$ or $\left.315^{\circ}\right), 80 \mathrm{~mm}$ from the starting position. The start of the trial was indicated by the appearance of the target. Subjects were instructed to 'shoot' through the target with a smooth arm movement as this type of movement is thought to rely on feed-forward control; in this type of movement, angular error at the start of movement is similar to the angular error at the end of movement suggesting that online feedback processes do not pay a major role in this task [13, 14]. The cursor was visible throughout the trial. If movement duration was greater than $300 \mathrm{~ms}$, the target changed from white to blue at the end of the trial indicating that the movement was too slow. After completion of the outward movement, participants were asked to relax and allow the robotic arm to return the arm to the central starting position. Once the cursor was re-centred the next target would appear.

Participants familiarised themselves with the basic task by performing 25 trials during which verbal feedback was given to further explain the desired movement (data not analysed). Each participant then completed five experimental conditions in which baseline performance was assessed and then subjects were examined for their ability to adapt and washout both visuomotor and forcefield perturbations (Fig. 1). The visuomotor condition consisted of a distortion of visual feedback by $30^{\circ}$ in the clockwise (positive) or anticlockwise (negative) direction. The forcefield condition consisted of a rightward (positive) or leftward (negative) velocity dependent force applied to the robotic arm during movement $(3 \mathrm{~N} /(\mathrm{m} / \mathrm{s})$ ). The type of adaptation perturbation was counterbalanced such that if the first perturbation was positive visuomotor, the second perturbation was negative forcefield (giving four possible order combinations). The total time of the experiment was approximately $45 \mathrm{~min}$.

\section{Kinematic Analysis}

Hand position was sampled at a rate of $200 \mathrm{~Hz}$. The outcome measures were angular error, movement duration and reaction time. Angular error was defined as the angular deviation from the ideal trajectory at the target perimeter. The start time $\left(t_{1}\right)$ of movement was defined as the time point at which $10 \%$ of maximal velocity of that trial was reached. This avoided
Fig. 1 a Overview of experimental design. Each epoch consisted of four trials. b Robotic apparatus and baseline task. Subjects were seated and held a manipulandum with their right hand (see text for full description). Upon appearance of the target they were trained to make a shooting movement through this as accurately as possible (the perfect path is indicated on the diagram by the dashed line). c Schematic drawing of the perturbation conditions. The visuomotor condition consisted of a distortion of visual feedback by $30^{\circ}$ in the clockwise (positive) or anticlockwise (negative) direction. The forcefield condition consisted of a rightward (positive) or leftward (negative) velocity dependent force applied to the robotic arm during movement $(3 \mathrm{~N} /(\mathrm{m} / \mathrm{s}))$
A

\begin{tabular}{|c|c|c|c|c|}
\hline $\begin{array}{l}\text { Baseline } \\
24 \text { epochs }\end{array}$ & $\begin{array}{l}\text { Adaptation } 1 \\
48 \text { epochs }\end{array}$ & $\begin{array}{l}\text { Washout } 1 \\
72 \text { epochs }\end{array}$ & $\begin{array}{c}\text { Adaptation } 2 \\
48 \text { epochs }\end{array}$ & $\begin{array}{l}\text { Washout } 2 \\
48 \text { epochs }\end{array}$ \\
\hline
\end{tabular}

B
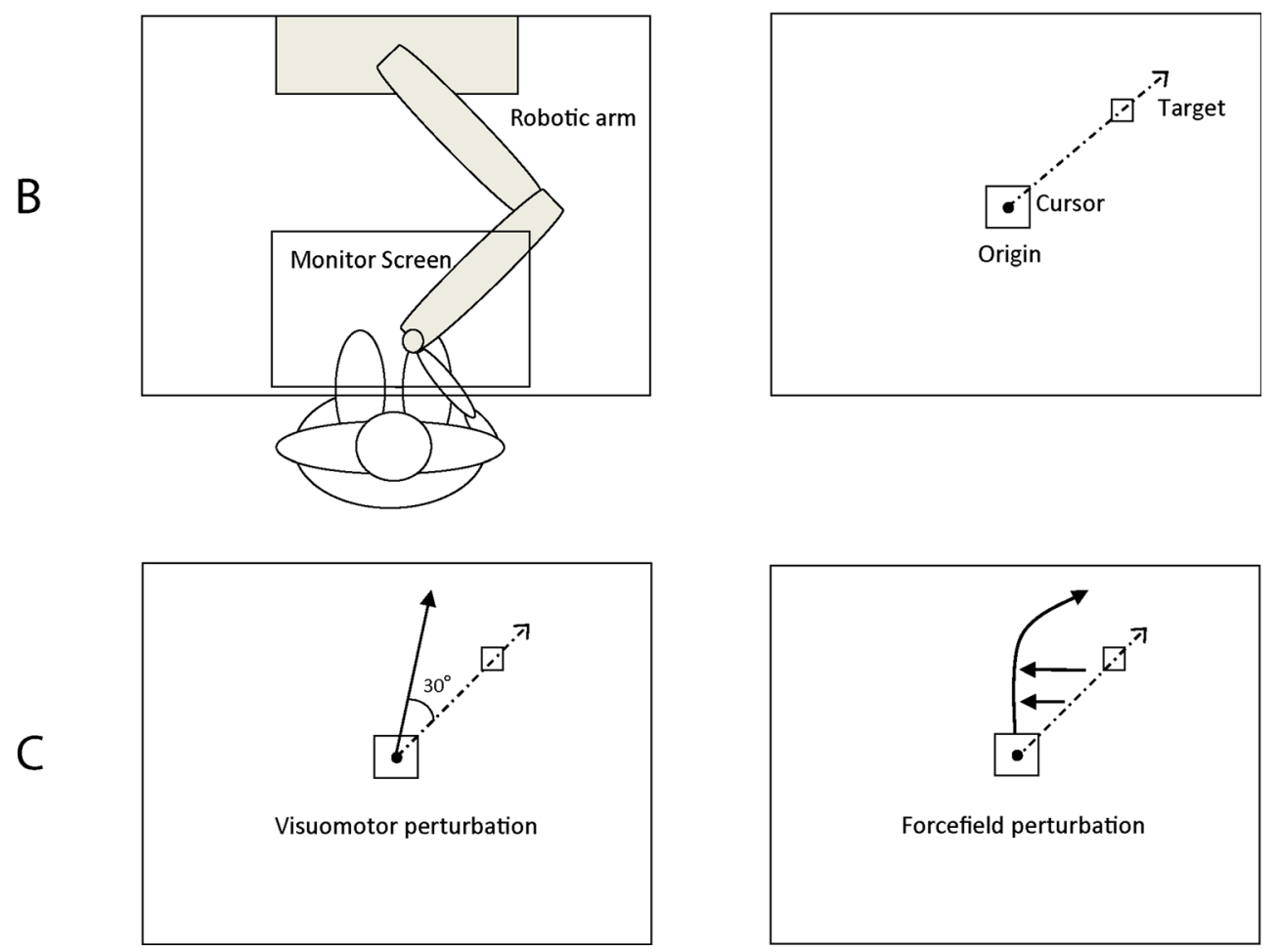
wrongly identifying small corrective movements of the cursor that were not the start of the shooting movement. The end of movement was defined as the time at which the target perimeter was first breached by subject movement $\left(t_{2}\right)$. Movement duration was the difference between these two values $\left(t_{2}-t_{1}\right)$. Reaction time was calculated as the difference between the time of target presentation $\left(t_{0}\right)$ and the start of movement $\left(t_{1}-t_{0}\right)$. Trials that had an angular error $> \pm 45^{\circ}$, a movement duration $<200 \mathrm{~ms}$ or $>800 \mathrm{~ms}$, or a reaction time $<200$ or $>600$, were excluded (in cervical dystonia $15.7 \%$ of trials, in controls $14.3 \%$ ). Epochs of all kinematic variables were created by taking an average value across four consecutive trials.

The primary outcome, angular error, of the four conditions (visuomotor adaptation, visuomotor washout, forcefield adaptation, forcefield washout) was modelled using:

$Y=a+\exp ^{(-c x)}$

where $Y$ represents the predicted angular error, $a$ is an estimate of the plateau of the learning curve, $b$ is an estimate of the maximal initial error (the $y$-intercept), $c$ estimates the learning index for each condition and $x$ is the epoch. The learning index is the percentage reduction in error for each epoch and thus can be used as a measure of the rate of adaptation and the rate of washout of perturbations. The adjusted $R^{2}$ value was calculated to analyse goodness of fit of the model. If $R^{2}$ was less than 0.4 (i.e., explained less than $40 \%$ of variation) then the individual's data for that perturbation were excluded from further group analysis (13\% excluded).

\section{Statistical Analysis}

SPSS (IBM SPSS Statistics, v21), Excel (Microsoft Excel for Mac 2011, v14.3.7) and Matlab (R2011b) were used for data analysis, and all data are given as mean \pm standard error of the mean (SEM). G*Power 3 [15] was used for the power calculation. Learning indices were compared using $t$ tests with Bonferonni correction for the four conditions (level of significance after correction 0.05/4). Reaction time and movement duration were compared between cervical dystonia and controls during the fast learning for each condition using analysis outlined in previous studies [14]. For each subject, a mean value was calculated during the initial rapid rate of learning such that for the baseline block (total of 24 epochs), epochs $2-$ 6 were averaged and for the adaptation and washout conditions (total of 48 epochs), epochs $2-11$ were averaged [16]. Repeated measures analysis of variance (rmANOVA) were used to compare mean reaction time with the factors GROUP (control, dystonia) and CONDITION (baseline, visuomotor adaptation, visuomotor washout, forcefield adaptation, forcefield washout). This analysis was repeated for movement duration.
The severity of cervical dystonia as defined by the TWSTRS (both severity subscore and total) and learning index were correlated (Pearson's correlation coefficient $(r)$ and the $p$ value are given). To examine for a potential relationship between tremor and adaptation, subjects were grouped into clinically apparent tremor and no tremor and $t$ tests were performed to compare the learning index of the two groups for the adaptation and washout conditions. For patients with clinically apparent tremor, total power as an estimate of severity was correlated to the learning index for each adaptation/washout condition. Log transformation of total power was performed to normalise data which allowed the subsequent Pearson's correlation.

\section{Results}

\section{Summary}

Rates of adaptation (learning) in cervical dystonia were identical to healthy controls in both visuomotor and forcefield tasks. Furthermore, the ability to adapt was not clearly related to clinical features of dystonic head tremor.

\section{Adaptation}

All subjects completed the experiments. Mean age and variability were matched between groups (control mean 56.0 years ( \pm 2.46$)$, patient mean 60.3 years $( \pm 2.80), t(36)=1.15, p=$ 0.255 ). One patient and one control were excluded from all further analysis due to consistently low movement durations (necessary due to velocity dependent forcefield). In addition, tremor data were not available (NA) for two patients due to a technical failure.

In Fig. 2, the angular error for the five conditions is shown for controls (red) and subjects with dystonia (blue). Visually, rates of learning were very similar between groups. To compare rates of learning, an exponential model was applied to each participant's data for the four adaptation conditions. In Fig. 3, the angular error of one subject (epochs of 4) is indicated by the solid grey line and the generated model by the dashed red line. It can be seen that the model accurately captures the slope of the curve in each condition which was the main parameter of interest (learning). To be included in analysis, the models adjusted $R^{2}$ had to exceed 0.4 . The number of exclusions is indicated in Table 2. Crucially, out of 152 models, $87 \%$ reached this criterion. The mean $R^{2}$ of the included models were not significantly different between groups: visuomotor adaptation $t(22.7)=-0.770, p=0.449$, visuomotor washout $t(33.7)=-0.525, p=0.603$, forcefield adaptation: $t(32)=0.413, p=0.683$, forcefield washout $t(25)=$ $0.187, p=0.853$. 


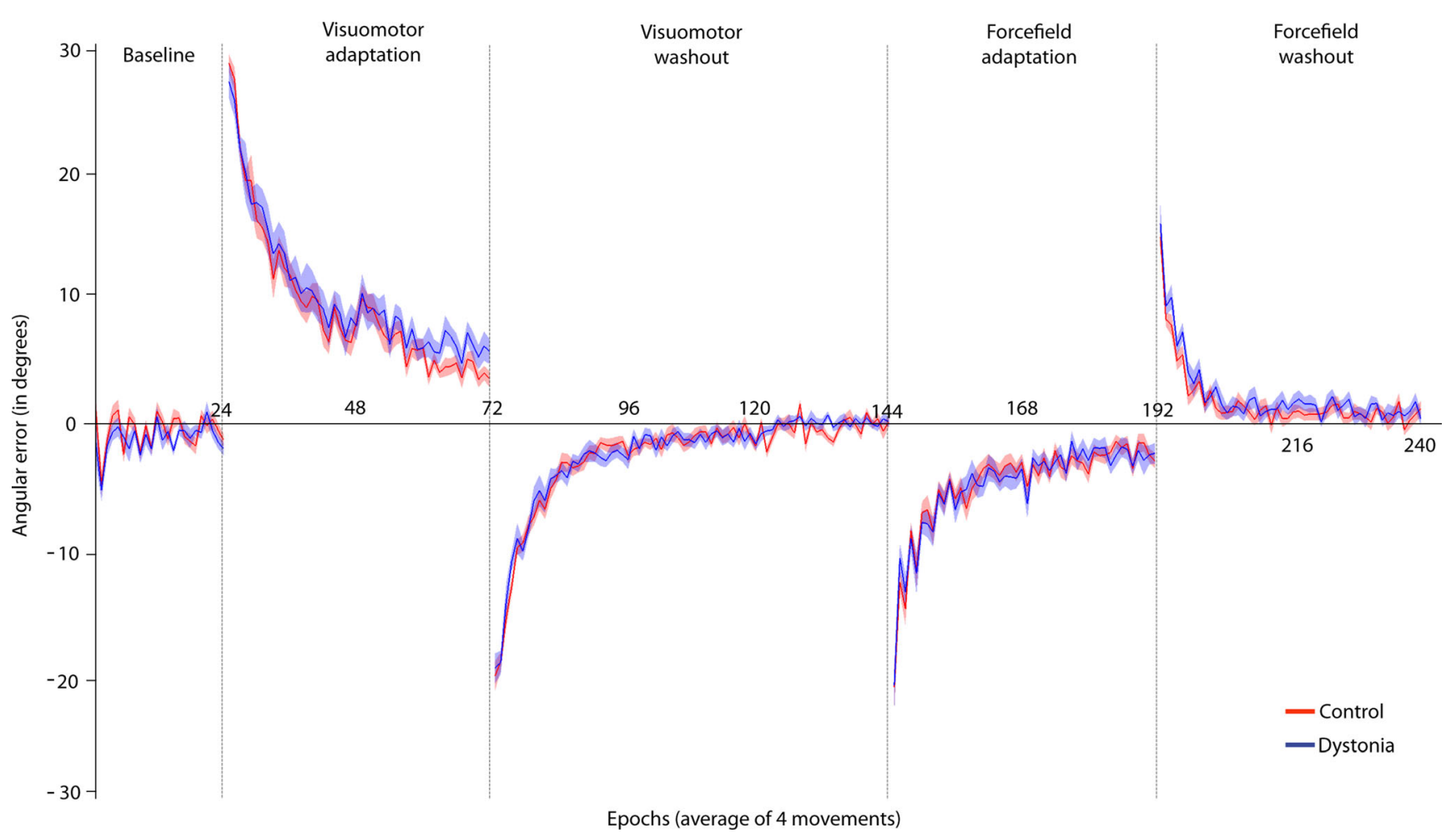

Fig. 2 Group data for angular error across baseline, adaptation and washout conditions. All data sorted into the same order for figure (experimental design consisted of four possible order combinations.) Control

The primary outcome, rate of adaptation and/or washout (mean learning indices) were not statistically different in any of the four conditions: visuomotor adaptation $t(33)=-0.396$, $p=0.695$, visuomotor washout $t(34)=0.287, p=0.776$, forcefield adaptation $t(32)=-0.553, p=0.584$ and forcefield washout $t(25)=0.254, p=0.801$. The profile was also remarkably similar in that for both groups (central bar chart Fig. 3): data shown in red; cervical dystonia data shown in blue. The solid line indicates the mean and the shaded regions the standard error

visuomotor adaptation was slower than forcefield adaptation as evidenced by smaller learning indices, washout of the visuomotor perturbations had a rate comparable to visuomotor adaptation and rates of forcefield washout were greater than the rates of forcefield adaptation. The plateau and maximal error for each condition were also similar in dystonia to controls (values given in Table 2, no statistical difference found).

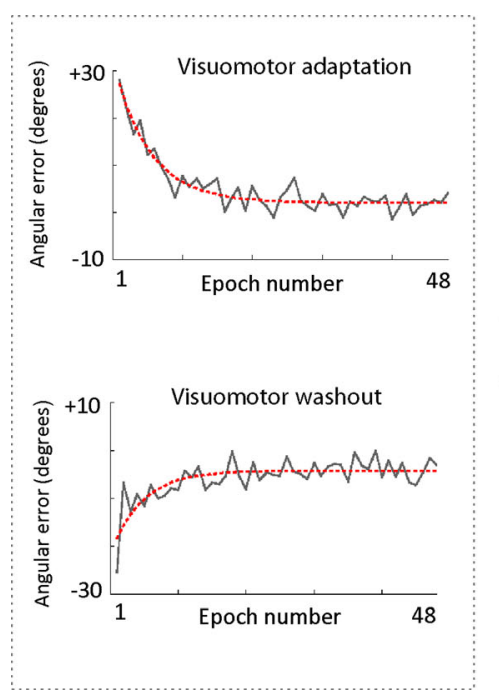

Fig. 3 The performance of the model for an individual patient, and its ability to capture the rate of learning for each condition, is shown in the boxes either side of the bar chart. The central bar chart plots mean
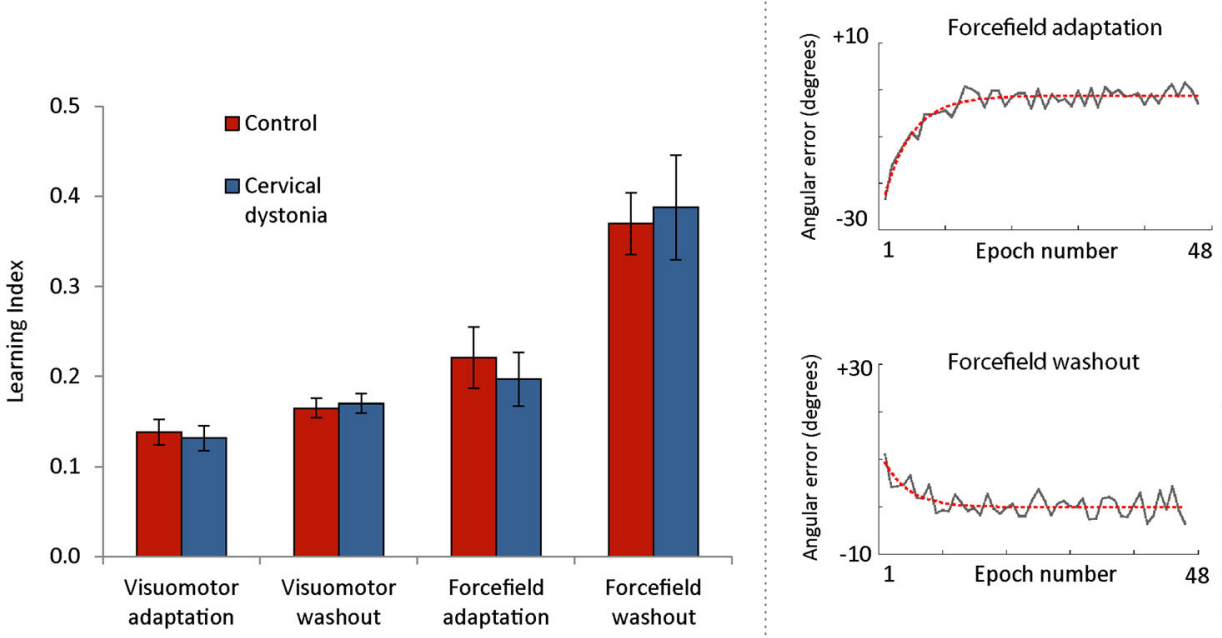

learning index for the two groups with the standard error of the mean indicated by the error bars. Red (left bar) control. Blue (right bar) cervical dystonia 


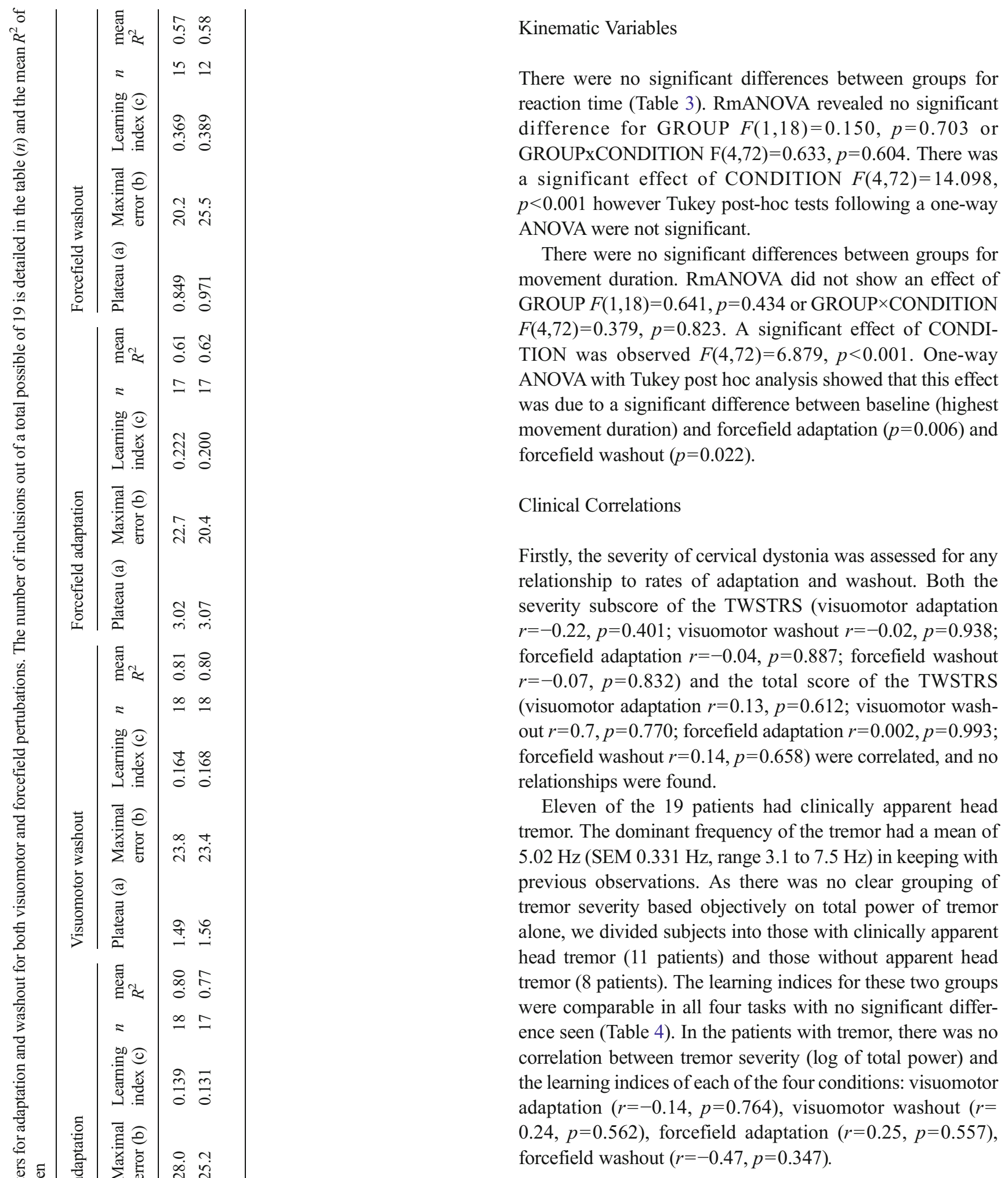

\section{Discussion}

In this study, we have demonstrated that motor adaptation in cervical dystonia is identical to healthy controls in two tasks 
Table 3 Mean reaction time and movement duration (with SEM) for patients and controls

\begin{tabular}{|c|c|c|c|c|}
\hline & \multicolumn{2}{|c|}{ Reaction time (ms) } & \multicolumn{2}{|c|}{ Movement duration (ms) } \\
\hline & Control & Cervical dystonia & Control & Cervical dystonia \\
\hline Baseline & $459(23)$ & $405(21)$ & $305(11)$ & $315(11)$ \\
\hline Visuomotor adaptation & $446(22)$ & 407 (16) & $290(7.7)$ & $289(9.2)$ \\
\hline Visuomotor washout & $414(20)$ & $413(18)$ & $288(6.4)$ & $291(8.3)$ \\
\hline Forcefield adaptation & $420(22)$ & $424(23)$ & $274(9.4)$ & $286(8.0)$ \\
\hline Forcefield washout & $387(17)$ & $439(24)$ & $278(7.1)$ & $289(6.8)$ \\
\hline
\end{tabular}

which test visual and proprioceptive sensorimotor integration. These data support preserved cerebellar function within this domain. We discuss these results in the context of recent dystonia research, which increasingly declares an important role for the cerebellum in the pathophysiology of dystonia.

\section{Evidence for Cerebellar Involvement in Dystonia}

Work in animal models strongly supports a causal cerebellar contribution in the genesis of dystonia. For example, in murine animal models, a dystonic-like condition can be provoked (excitation) or eliminated (inhibition or cerebellectomy) by modulating the activity of the cerebellum [17] and genetically modified animal models are increasingly sophisticated in their ability to probe and implicate the cerebellum [18].

In humans, clinical data suggests that structural and degenerative disorders of the cerebellum can cause secondary forms of dystonia [19]. However, secondary dystonia, by definition, has differences to primary dystonia in which no gross structural pathology is observed. In addition, such disorders rarely selectively involve only the cerebellum, and pathology within the cerebellum may evoke compensatory change in a multitude of interconnected regions. Therefore, parallels drawn between this clinical data and primary dystonia should be tentative. In primary dystonia, subtle structural and functional abnormalities of the cerebellum and its communicating tracts have been consistently demonstrated with a range of imaging techniques (for review see [20-22]). Some electrophysiological data also point to cerebellar abnormalities in dystonia. Cerebellar brain inhibition (CBI) of the motor cortex was reduced in a small number of patients with focal hand dystonia [23]. In addition, the ability to acquire eye blink conditioning was reduced in cervical and focal hand dystonia, suggesting impaired cerebellar function [24]. Interestingly, this deficit in conditioning could be improved by further practice or inhibitory cerebellar stimulation, suggesting that cerebellar 'dysfunction' in dystonia is a dynamic process [25]. To date, deficits in CBI have not been replicated in a larger study nor investigated in other subgroups of dystonia and we have recently shown that eye blink conditioning is intact in the genetic dystonias DYT1 and DYT6 (submitted). Another line of investigation examining cerebellar function in dystonia has been the study of motor tasks which require intact cerebellar function. In DYT1 dystonia, both manifesting and nonmanifesting subjects are impaired in sequence learning in which the sequential order targets is learnt $[26,27]$ and functional imaging demonstrated overactivity of the left cerebellar cortex (whilst subjects moved the right arm) [27, 28]. However, sequence learning recruits many brain regions including the basal ganglia, and the overactivation of the contralateral cerebellar hemisphere to hand movement (cerebellar control is ipsilateral) makes the functional significance of these findings in dystonia difficult to elucidate. Recently, it has been shown that sequence learning is normal in cervical dystonia [29].

\section{Motor Adaptation and Dystonia}

Motor adaptation is a task commonly used, across species, to directly examine cerebellar function [14, 30]. An environmental perturbation introduces a movement error requiring
Table 4 Comparison of the mean values of learning indices in patients with and without tremor. Learning indices were only included in comparison if the model suitably fitted the data $\left(R^{2}>0.4\right)$. Eleven of the 19 patients had clinically apparent tremor

\begin{tabular}{lllll}
\hline Condition & Group & $n$ & Learning index mean (SEM) & $t$ test \\
\hline Visuomotor adaptation & No tremor & 8 & $0.108(0.019)$ & $t(15)=1.58, p=0.134$ \\
& Tremor & 9 & $0.152(0.021)$ & \\
Visuomotor washout & No tremor & 8 & $0.182(0.018)$ & $t(16)=-1.18, p=0.280$ \\
& Tremor & 10 & $0.158(0.013)$ & $t(15)=0.188, p=0.853$ \\
Forcefield adaptation & No tremor & 7 & $0.189(0.049)$ & $t(10)=-0.255, p=0.804$ \\
& Tremor & 10 & $0.201(0.042)$ & \\
\hline \multirow{3}{*}{ Forcefield washout } & No tremor & 5 & $0.412(0.048)$ & \\
& Tremor & 7 & $0.373(0.125)$ &
\end{tabular}


subjects to adapt their performance of a task. The sensory prediction error (how the actual sensory movement outcome differed from the predicted sensory movement outcome) is used to update subsequent motor performance, with this type of learning being strongly dependent on the cerebellum [5]. Interestingly, the cerebellum has not only been linked to the formation of forward models which predict the sensory outcomes of motor commands; it may be that the cerebellum has a role in forming cognitive predictions for non-motor cerebellar functions such as language [31,32]. This argument is supported by the highly conserved structure of the cerebellar microanatomical architecture, which is thought to imply that the computational qualities of cerebellar cortex remain constant [32, 33].

Patients with myoclonus dystonia (caused by mutations of the SGCE gene, DYT11) have been shown to have impaired saccadic adaptation [34]. It is difficult to dissociate in this condition whether the predominant phenotype of myoclonus dominates the neuroanatomical findings or whether the milder dystonia also has a role. In primary dystonia, motor learning/ adaptation has been examined in focal hand dystonia using a joystick task [35]. Each trial had a different visuomotor perturbation, and a different position of the target and subjects were asked to correct their movement during each trial. No impairment in motor learning was demonstrated but there was impaired retention. However, contrary to the author's conclusions, this suggests a change in the ability of the motor cortex to retain the new memory rather than a cerebellar deficit [14, 35]. Our data in cervical dystonia builds on previous work that we performed with a more simplistic visuomotor adaptation task [29]. This current study differs in that we used a purpose built robot which required larger more complex movements recruiting proximal arm and shoulder muscles. We also used a shooting paradigm which does not allow for online correction and modelled data in a manner which we believe optimally assesses for differences in adaptation. The forcefield condition is more relevant to dystonia in which subtle proprioceptive deficits have been described [36]. Furthermore, visuomotor and forcefield adaptation examine distinct (and common) regions of cerebellar function. Within the anterior lobe of the cerebellum, which contains one of the two body representations within the cerebellum, lobules IV and V are thought to be more important for the forcefield task and lobule VI is more important for visuomotor adaptation [8]. Regions in the posterolateral cerebellum (crus I and II) are thought to be required for both tasks [22]. This analysis of the two perturbations with a large number of patients leads us to confidently conclude that motor adaptation is normal in cervical dystonia.

\section{Significance of our Results}

How do our results link in with the growing body of evidence which implicates the cerebellum in the pathophysiology of dystonia? Certainly for cervical dystonia, if there is cerebellar dysfunction, the nature and extent of cerebellar dysfunction remain to be established. Normal cerebellar adaptation in cervical dystonia is in contrast to deficits in eye blink conditioning within the same subtype of dystonia [24, 25]. Both paradigms are well-characterised paradigms in their assessment of cerebellar function. Perhaps the deficit in eye blink conditioning, with its greater reliance on millisecond timing, signifies that timing is the specific cerebellar deficit in dystonia? This viewpoint links well with abnormalities in temporal discrimination and other timing tasks that have been found within the millisecond range in focal dystonia [37-39]. One counter to this argument is the observation that all movement parameters were normal in the current study, and each parameter has a millisecond timing requirement. Furthermore, a deficit in millisecond timing capabilities could potentially impair the generation of sensory prediction errors, which would include time as one of their dimensions.

The normal performance in these adaptation tasks that required use of both visual and proprioceptive input was of interest. Although visual processing is normal in cervical dystonia, previous studies have described deficits in proprioceptive tasks dystonic subjects are less sensitive at detecting passive movements of the fingers [40] and arms are abnormal in their perception of the vibration induced illusion of movement (which is induced by stimulating muscle spindles with a vibration stimulus) [36, 41, 42]. How can performance in our tasks be normal in the face of such obvious deficits? One possibility is that tests of proprioceptive sensation are mostly static tasks whereas ours were dynamic, involving sensation during active movement. Furthermore, the psychophysical tasks described above require sensory processing and decision making at many levels of the nervous system and some of these are likely to be distinct to networks involved in implicit motor tasks. For example, higher order/consciously regulated elements of decision making could have a greater influence on psychophysical tasks.

The question of whether movement in the asymptomatic arm of patients with cervical dystonia is entirely normal perhaps remains to be definitively answered with future experimental work. Some have described abnormalities in kinematic variables recorded during reaching studies similar to the task used in this article (movement time was not matched between groups and thus some of this data is difficult to interpret [40]) and electrophysiologically, abnormalities in inhibition have been demonstrated at many levels of the nervous system concerned with the control of the arm musculature (e.g., abnormal reciprocal inhibition of forearm muscles in cervical dystonia [43]). However, other studies in including ours suggest near normal motor performance [29]. Conservation of motor skill in the arms is the norm with most patients with cervical dystonia, and we argue that this is perhaps against a global movement deficit in the focal dystonias. 
Our conclusions for dystonic tremor are more tentative. We did not find evidence to support a relationship between the ability to adapt and the severity of dystonic tremor. Secondly, splitting subjects into whether or not they had tremor did not reveal a group difference in rates of adaptation. The pathophysiology of dystonic tremor is poorly understood but many primary tremor models are thought to involve the cerebellothalamocortical network. Certainly, in patients with essential tremor, there seems to be multimodal evidence for pathological involvement of the cerebellum (structural imaging [44], functional imaging [45], eye movement analysis [46], deficits in eye blink conditioning [47] and motor adaptation [12].) Here, we have performed one of the first studies to examine the role of the cerebellum in the generation of dystonic head tremor and have not yet found a clear interaction. Our findings support studies that suggest different mechanisms between essential and dystonic tremor. For example, in essential tremor, the second agonist burst during ballistic movements is delayed and this finding is often ascribed to a lack of cerebellar prediction [48]. This delay in timing is not observed in patients with dystonic tremor [49].

A final implication of our results is that the preservation of adaptation, a type of motor learning, may have potential therapeutic implications. Adaptation could be used to reduce errors in dystonic movements, and this could translate into advances in physical therapy for dystonia [50].

A limitation of our study is the possibility that our task was insensitive to a deficit in adaptation. Perhaps errors were too large in our task to detect cerebellar dysfunction within a biologically relevant range. Against this is the observation that patients with cerebellar damage had an equal difficulty with small and large perturbation errors [51]. Furthermore, based on our mean and variance from the visuomotor adaptation condition (effect size 0.097 ) and assuming a power level of 0.8 , we would need approximately 2,700 subjects in total in order to achieve a significant result. Therefore, we do not believe our null results are due to a lack of power. Another perhaps unavoidable limitation is that patients were receiving botulinum toxin injections (the mainstay of treatment for cervical dystonia). We tested patients when maximally symptomatic prior to injections but the long-term influence of botulinum injections on results cannot not be fully assessed in this or other studies that have used an identical approach.

\section{Conclusions}

We have shown that adaptation, a fundamental computation of the cerebellum, is normal in cervical dystonia. Furthermore, the ability to adapt is not clearly related to clinical features of dystonic head tremor. These results have important implications in the current thinking of the pathophysiology of dystonia. It is important to progress from very general statements that implicate the cerebellum in the genesis of dystonia to a more specific evidence-based model. Future research should aim to critically and directly examine the extent and nature of cerebellar involvement in a hypothesis driven framework. The role of the cerebellum in this enigmatic disease perhaps remains to be proven.

Acknowledgments We would like to thank the patients and control subjects that gave their time to participate in this study.

Conflicts of interest Sadnicka A was supported by a grant from the Guarantors of Brain, UK. Saifee TA was supported by a fellowship awarded by the National Institute for Health Research (UK). Kassavetis $\mathrm{P}$ was supported by a grant from Parkinson's UK. Pareés I was funded by a EFNS scientific fellowship. Korlipara LVP has received funding for travel and to attend meetings from TEVA, Genus Pharmaceuticals and Glaxo Smithkline. He has received speaker honoraria from Glaxo Smithkline. Bhatia KP received funding for travel from GlaxoSmithKline, Orion Corporation, Ipsen and Merz Pharmaceuticals, LLC; serves on the editorial boards of Movement Disorders and Therapeutic Advances in Neurological Disorders; receives royalties from the publication of Oxford Specialist Handbook of Parkinson's Disease and Other Movement Disorders (Oxford University Press, 2008); received speaker honoraria from GlaxoSmithKline, Ipsen, Merz Pharmaceuticals, LLC, and Sun Pharmaceutical Industries Ltd.; personal compensation for scientific advisory board for GSK and Boehringer Ingelheim; received research support from Ipsen and from the Halley Stewart Trust through Dystonia Society UK, and the Wellcome Trust MRC strategic neurodegenerative disease initiative award (Ref. number WT089698), a grant from the Dystonia Coalition and a grant from Parkinson's UK (Ref. number G-1009). Rothwell JC has received speaker travel costs from the Movement Disorders Society. Edwards MJ receives royalties from publication of Oxford Specialist Handbook of Parkinson's Disease and Other Movement Disorders (Oxford University Press, 2008) and receives research support from a National Institute for Health Research (NIHR) grant where he is the PI. He has received honoraria for speaking from UCB. Patani B and Galea JM have no conflict of interest to declare.

Open Access This article is distributed under the terms of the Creative Commons Attribution License which permits any use, distribution, and reproduction in any medium, provided the original author(s) and the source are credited.

\section{References}

1. Frucht SJ. The definition of dystonia: current concepts and controversies. Mov Disord. 2013;28:884-8.

2. Albanese A, Bhatia K, Bressman SB, Delong MR, Fahn S, Fung VS, et al. Phenomenology and classification of dystonia: a consensus update. Mov Disord. 2013;28:863-73.

3. Sadnicka A, Hoffland BS, Bhatia KP, van de Warrenburg BP, Edwards MJ. The cerebellum in dystonia - help or hindrance? Clin Neurophysiol. 2012;123:65-70.

4. Filip P, Lungu OV, Bares M. Dystonia and the cerebellum: a new field of interest in movement disorders? Clin Neurophysiol. 2013;124:1269-76.

5. Krakauer JW, Mazzoni P. Human sensorimotor learning: adaptation, skill, and beyond. Curr Opin Neurobiol. 2011;21:636-44.

6. Maschke M, Gomez CM, Ebner TJ, Konczak J. Hereditary cerebellar ataxia progressively impairs force adaptation during goal-directed arm movements. J Neurophysiol. 2004;91:230-8. 
7. Martin TA, Keating JG, Goodkin HP, Bastian AJ, Thach WT. Throwing while looking through prisms. I. Focal olivocerebellar lesions impair adaptation. Brain. 1996;119(Pt 4):1183-98.

8. Donchin O, Rabe K, Diedrichsen J, Lally N, Schoch B, Gizewski ER, et al. Cerebellar regions involved in adaptation to force field and visuomotor perturbation. J Neurophysiol. 2012;107:134-47.

9. Shadmehr R, Smith MA, Krakauer JW. Error correction, sensory prediction, and adaptation in motor control. Annu Rev Neurosci. 2010;33:89-108.

10. Krakauer JW, Pine ZM, Ghilardi MF, Ghez C. Learning of visuomotor transformations for vectorial planning of reaching trajectories. J Neurosci. 2000;20:8916-24.

11. Hwang EJ, Smith MA, Shadmehr R. Adaptation and generalization in acceleration-dependent force fields. Exp Brain Res. 2006;169:496-506.

12. Chen H, Hua SE, Smith MA, Lenz FA, Shadmehr R. Effects of human cerebellar thalamus disruption on adaptive control of reaching. Cereb Cortex. 2006;16:1462-73.

13. Tseng YW, Diedrichsen J, Krakauer JW, Shadmehr R, Bastian AJ. Sensory prediction errors drive cerebellum-dependent adaptation of reaching. J Neurophysiol. 2007;98:54-62.

14. Galea JM, Vazquez A, Pasricha N, de Xivry JJ, Celnik P. Dissociating the roles of the cerebellum and motor cortex during adaptive learning: the motor cortex retains what the cerebellum learns. Cereb Cortex. 2011;21:1761-70

15. Faul F, Erdfelder E, Lang AG, Buchner A. G*Power 3: a flexible statistical power analysis program for the social, behavioral, and biomedical sciences. Behav Res Methods. 2007;39:175-91.

16. Krakauer JW, Ghez C, Ghilardi MF. Adaptation to visuomotor transformations: consolidation, interference, and forgetting. J Neurosci. 2005;25:473-8.

17. Wilson BK, Hess EJ. Animal models for dystonia. Mov Disord. 2013;28:982-9.

18. Oleas J, Yokoi F, Deandrade MP, Pisani A, Li Y. Engineering animal models of dystonia. Mov Disord. 2013;28:990-1000.

19. Fletcher NA, Stell R, Harding AE, Marsden CD. Degenerative cerebellar ataxia and focal dystonia. Mov Disord. 1988;3:336-42.

20. Lerner RP, Niethammer M, Eidelberg D. Understanding the anatomy of dystonia: determinants of penetrance and phenotype. Curr Neurol Neurosci Rep. 2013;13:401.

21. Niethammer M, Carbon M, Argyelan M, Eidelberg D. Hereditary dystonia as a neurodevelopmental circuit disorder: evidence from neuroimaging. Neurobiol Dis. 2011;42:202-9.

22. Neychev VK, Gross RE, Lehericy S, Hess EJ, Jinnah HA. The functional neuroanatomy of dystonia. Neurobiol Dis. 2011;42:185-201.

23. Brighina F, Romano M, Giglia G, Saia V, Puma A, Giglia F, et al. Effects of cerebellar TMS on motor cortex of patients with focal dystonia: a preliminary report. Exp Brain Res. 2009;192:651-6.

24. Teo JT, van de Warrenburg BP, Schneider SA, Rothwell JC, Bhatia KP. Neurophysiological evidence for cerebellar dysfunction in primary focal dystonia. J Neurol Neurosurg Psychiatry. 2009;80:80-3.

25. Hoffland BS, Kassavetis P, Bologna M, Teo JT, Bhatia KP, Rothwell JC, et al. Cerebellum-dependent associative learning deficits in primary dystonia are normalized by rTMS and practice. Eur J Neurosci. 2013;38:2166-71.

26. Ghilardi MF, Carbon M, Silvestri G, Dhawan V, Tagliati M, Bressman S, et al. Impaired sequence learning in carriers of the DYT1 dystonia mutation. Ann Neurol. 2003;54:102-9.

27. Carbon M, Ghilardi MF, Argyelan M, Dhawan V, Bressman SB, Eidelberg D. Increased cerebellar activation during sequence learning in DYT1 carriers: an equiperformance study. Brain. 2008;131:146-54.

28. Carbon M, Argyelan M, Ghilardi MF, Mattis P, Dhawan V, Bressman $\mathrm{S}$, et al. Impaired sequence learning in dystonia mutation carriers: a genotypic effect. Brain. 2011;134:1416-27.

29. Katschnig-Winter P, Schwingenschuh P, Davare M, Sadnicka A, Schmidt R, Rothwell JC, Bhatia KP, Edwards MJ: Motor sequence learning and motor adaptation in primary cervical dystonia. J Clin Neurosci 2013.
30. Jayaram G, Galea JM, Bastian AJ, Celnik P. Human locomotor adaptive learning is proportional to depression of cerebellar excitability. Cereb Cortex. 2011;21:1901-9.

31. Lesage E, Morgan BE, Olson AC, Meyer AS, Miall RC. Cerebellar rTMS disrupts predictive language processing. Curr Biol. 2012;22: R794-5.

32. Dean P, Porrill J. The cerebellum as an adaptive filter: a general model? Funct Neurol. 2010;25:173-80.

33. Dean P, Porrill J. Evaluating the adaptive-filter model of the cerebellum. J Physiol. 2011;589:3459-70.

34. Hubsch C, Vidailhet M, Rivaud-Pechoux S, Pouget P, Brochard V, Degos B, et al. Impaired saccadic adaptation in DYT11 dystonia. J Neurol Neurosurg Psychiatry. 2011;82:1103-6.

35. Hubsch H, Roze E, Popa T, Russo M, Balachandran A, Pradeep S, et al. Defective cerebellar control of cortical plasticity in writer's cramp. Brain. 2013;136:13.

36. Frima N, Nasir J, Grunewald RA. Abnormal vibration-induced illusion of movement in idiopathic focal dystonia: an endophenotypic marker? Mov Disord. 2008;23:373-7.

37. Filip P, Lungu OV, Shaw DJ, Kasparek T, Bares M. The mechanisms of movement control and time estimation in cervical dystonia patients. Neural Plast. 2013;2013:908741.

38. Hutchinson M, Kimmich O, Molloy A, Whelan R, Molloy F, Lynch T, et al. The endophenotype and the phenotype: temporal discrimination and adult-onset dystonia. Mov Disord. 2013;28: $1766-74$.

39. Avanzino L, Martino D, Martino I, Pelosin E, Vicario CM, Bove M, et al. Temporal expectation in focal hand dystonia. Brain. 2013;136: 444-54.

40. Putzki N, Stude P, Konczak J, Graf K, Diener HC, Maschke M. Kinesthesia is impaired in focal dystonia. Mov Disord. 2006;21:754 60.

41. Rome S, Grunewald RA. Abnormal perception of vibration-induced illusion of movement in dystonia. Neurology. 1999;53:1794-800.

42. Yoneda Y, Rome S, Sagar HJ, Grunewald RA. Abnormal perception of the tonic vibration reflex in idiopathic focal dystonia. Eur J Neurol. 2000;7:529-33.

43. Deuschl G, Seifert C, Heinen F, Illert M, Lucking CH. Reciprocal inhibition of forearm flexor muscles in spasmodic torticollis. J Neurol Sci. 1992;113:85-90.

44. Shin DH, Han BS, Kim HS, Lee PH. Diffusion tensor imaging in patients with essential tremor. AJNR Am J Neuroradiol. 2008;29:151-3.

45. Jenkins IH, Bain PG, Colebatch JG, Thompson PD, Findley LJ, Frackowiak RS, et al. A positron emission tomography study of essential tremor: evidence for overactivity of cerebellar connections. Ann Neurol. 1993;34:82-90.

46. Helmchen C, Hagenow A, Miesner J, Sprenger A, Rambold H, Wenzelburger R, et al. Eye movement abnormalities in essential tremor may indicate cerebellar dysfunction. Brain. 2003;126:1319-32.

47. Kronenbuerger M, Gerwig M, Brol B, Block F, Timmann D. Eyeblink conditioning is impaired in subjects with essential tremor. Brain. 2007;130:1538-51.

48. Munchau A, Schrag A, Chuang C, MacKinnon CD, Bhatia KP, Quinn NP, et al. Arm tremor in cervical dystonia differs from essential tremor and can be classified by onset age and spread of symptoms. Brain. 2001;124:1765-76.

49. Koster B, Deuschl G, Lauk M, Timmer J, Guschlbauer B, Lucking $\mathrm{CH}$. Essential tremor and cerebellar dysfunction: abnormal ballistic movements. J Neurol Neurosurg Psychiatry. 2002;73:400-5.

50. van den Dool J, Visser B, Koelman JH, Engelbert RH, Tijssen MA. Cervical dystonia: effectiveness of a standardized physical therapy program; study design and protocol of a single blind randomized controlled trial. BMC Neurol. 2013;13:85.

51. Schlerf J, Ivry RB, Diedrichsen J. Encoding of sensory prediction errors in the human cerebellum. J Neurosci. 2012;32:4913-22. 